\title{
L'ART PLUS FORT QUE LA SCIENCE? \\ L'affrontement entre expertise stylistique et expertise scientifique dans une querelle sur l'authenticité d'une œuvre d'art: l'affaire Sésostris III
}

\section{Alain Quemin*}

\begin{abstract}
Résumé: L'une des dimensions de l'expertise artistique consiste à se prononcer sur l'authenticité des œuvres et, pour cela, les experts disposent de différentes méthodes et ressources. Si, traditionnellement, l'essentiel des arguments relevait du registre stylistique, de la manière de procéder qui permettait d'attribuer telle ou telle œuvre à un artiste, de nouvelles méthodes scientifiques (tests chimiques, de thermoluminescence...) fondées sur l'étude des propriétés physiques des œuvres, ont, depuis, connu un développement considérable. Le présent texte se propose d'analyser un cas exemplaire de controverse liée à l'authenticité d'une œuvre, une statue du pharaon égyptien Sésostris III, qui a vu s'affronter arguments stylistiques et arguments scientifiques (entendus ici au sens de sciences "dures") et d'étudier comment des arguments opposés ont été reçus par des tierces parties comme la Justice, parfois amenée in fine à trancher les cas les plus épineux.
\end{abstract}

Mots clefs: expertise, controverse art-science, droit.

Le présent texte se propose d'analyser un cas exemplaire de controverse liée à l'authenticité d'une œuvre qui a vu s'affronter arguments stylistiques et arguments scientifiques (entendus ici au sens de sciences “dures") et d'étudier comment des arguments opposés ont été reçus par des tierces parties comme la Justice, parfois amenée in fine à trancher les cas les plus épineux. A partir de

* Professor de Sociologia, Université de Marne-la-Vallée / Institut Universitaire de France, LATTS (CNRS). E-mail: quemin@univ-mlv.fr

Artigo recebido em 16 mai. 2005; aprovado em 22 jun. 2005. 
l'exemple d'une statue du pharaon égyptien Sésostris III acquise par le milliardaire français et collectionneur d'art François Pinault, nous ferons apparaître qu'au final, et même si ceci peut sembler à première vue paradoxal, ce sont bien souvent les analyses artistiques, stylistiques qui l'emportent, quand bien même les tests scientifiques indiquent que les œuvres qui font l'objet de débats sont des faux. Alors même que la science entraîne fréquemment l'adhésion dans de nombreux domaines de la vie sociale, il importera de s'interroger sur le crédit considérable dont continuent à bénéficier les experts tels que conservateurs de musée et historiens d'art dans le champ artistique, et sur la mise à distance des méthodes scientifiques pour déterminer si une œuvre est bien authentique ou non, dans les cas où savoir artistique et savoir scientifique apparaissent en rivalité.

L'une des dimensions de l'expertise artistique consiste à se prononcer sur l'authenticité des œuvres et, pour cela, les experts disposent de différentes méthodes et ressources (Moulin et Quemin, 1993, p. 1421-1445).

$\mathrm{Si}$, traditionnellement, l'essentiel des arguments relevait du registre stylistique, de la manière de procéder qui permettait d'attribuer telle ou telle œuvre à un artiste, de nouvelles méthodes scientifiques ont, depuis, connu un développement considérable. Désormais, en effet, l'utilisation de certaines technologies (tests chimiques, de thermoluminescence...) permet d'émettre un avis en se fondant sur des propriétés physiques des œuvres.

Le présent texte se propose d'analyser - sous une forme encore inachevée - un cas emblématique de controverse liée à l'authenticité d'une œuvre qui a vu s'affronter arguments stylistiques et arguments scientifiques (entendus ici au sens de sciences "dures") et d'étudier comment des arguments opposés ont été reçus par des tierces parties comme la Justice, comme souvent amenée in fine à trancher les cas les plus épineux. Cela permet d'illustrer la rencontre de trois mondes sociaux généralement étanches les uns aux autres, celui de l'art (cf. Becker, 1988), celui des sciences et celui du droit. ${ }^{1}$ A partir du célèbre cas de la statue du pharaon Sésostris III, nous ferons apparaître qu'au final, et même si ceci peut sembler à première 
vue paradoxal, ce sont parfois les analyses artistiques, stylistiques, qui l'emportent, quand bien même les tests «scientifiques» indiquent que les œuvres qui font l'objet de débats sont des faux.

Alors même que la science entraîne fréquemment l'adhésion dans de nombreux domaines de la vie sociale, il importera de s'interroger sur le crédit considérable dont continuent à bénéficier les experts tels que conservateurs de musée et historiens d'art dans le champ artistique, mais aussi dans celui de la justice, et sur la mise à distance des méthodes scientifiques pour déterminer si une œuvre est bien authentique ou non, dans les cas où savoir artistique et savoir scientifique apparaissent en rivalité.

Une remarquable narration des débuts de l'affaire Sésostris III jusqu'à l' année 2002 est rapportée par Vincent Noce, le journaliste ayant couvert l'affaire pour Libération, dans son ouvrage Descente aux enchères: les coulisses du marché de l'art (2002).

Le 16 novembre 1998, le célèbre homme d'affaires François Pinault et son épouse Maryvonne apprenaient, à la lecture du quotidien Libération, qu'au terme de l'enquête menée par Vincent Noce, une statue du pharaon Sésostris III, considérée comme vieille de 3800 ans qu'ils avaient achetée une semaine plus tôt à Paris, le 10 novembre 1998, pour la coquette somme de 5,1 millions de francs (près de 800.000 euros) - un record pour une pièce archéologique en France - pouvait n'être en réalité qu'un faux assez grossier fabriqué au $\mathrm{XIX}^{\text {ème }}$ siècle ou même au $\mathrm{XX}^{\text {ème }}$ siècle.

Telle était la conviction de l'éminent spécialiste Dietrich Wildung, directeur du musée de l'antiquité égyptienne de Berlin, dont les propos étaient rapportés, et qui précisait avoir alerté en vain les responsables de l'hôtel Drouot avant la vente. Et le conservateur allemand de préciser que la pièce lui avait été présentée une quinzaine d'années auparavant mais que: Nous avons refusé de l'acheter, car j'avais de sérieuses raisons de penser qu'elle aurait été fabriquée dans la première moitié du Xxème siècle (après Jésus-Christ).

L'affaire qui éclatait ainsi était d'autant plus gênante que d'aucuns prétendaient que Madame Pinault avait acheté la statue du 
pharaon dans l'optique de la remettre un jour au musée du Louvre, les époux Pinault faisant partie de ces riches collectionneurs que les musées français prennent soin d'associer à leur politique d'acquisitions. En privé, l'expert de la vente confiait d'ailleurs avoir reçu un avis très favorable d'un conservateur qui avait pu examiner de près et admirer l'œuvre en question. Ceci ne pouvait toutefois faire l'objet d'un avis officiel, les conservateurs de musées étant tenus, en France, à un devoir de réserve pour les pièces présentées sur le marché (Moulin et Quemin, 1993, p. 1421-1445).

La statue du pharaon Sésostris III constituait le premier achat d'une antiquité de cette importance par les époux Pinault, François Pinault étant davantage amateur d'art contemporain et son épouse d'œuvres d'art du XVIII ${ }^{\text {ème }}$ siècle tout particulièrement. Leur jugement d'amateurs pouvait ainsi se trouver terni et c'est sans doute pourquoi leur premier réflexe fut de faire démentir par l'agence de relations publiques de François Pinault que le couple puisse être l'acquéreur de la statue désormais contestée. En même temps, François Pinault faisait aussitôt suspendre le paiement du commissaire-priseur, Maître Olivier Couteau-Bégarie, auprès duquel il avait acquis la statue controversée, et il réclamait des éclaircissements au professionnel. Devant le caractère peu convaincant, à ses yeux, des explications fournies, il demandait l'annulation de la vente au commissaire-priseur. ${ }^{2}$

Les époux Pinault ayant demandé l'annulation de la vente, il leur revenait désormais de faire la preuve que la statue devenue suspecte constituait bien un faux. Un problème de taille se posait alors : s'il est aisé de dater les œuvres en terre cuite de façon fiable et précise à l'aide de tests scientifiques, on ne dispose guère de méthodes scientifiques permettant d'établir de façon aussi rigoureuse la date d'une sculpture de pierre - ici, de granit - comme celle du pharaon Sésostris III. L'argumentation permettant de contester l'authenticité de l'œuvre devait donc se porter tout d'abord sur le terrain de l'analyse stylistique.

C'est bien sur la base de tels arguments que le célèbre égyptologue allemand Dietrich Wildung a entrepris de prouver que 
l'œuvre constituait incontestablement un faux, assez grossier de surcroît. Pour ce spécialiste, l'œuvre constituait «un ensemble de maladresses» qu'aucun artisan d'atelier royal n'aurait pu commettre. En termes strictement stylistiques, la statue présentait, selon lui, des jambes trop longues, une coiffure disymétrique, mais elle présentait également des anachronismes à travers des analogies avec un style ayant bien existé en Egypte, mais postérieurement à la mort du souverain. Dietrich Wildung connaissait d'autant mieux la statue que celle-ci avait été proposée à la vente à son musée en 1980, mais qu'il avait repoussé l'offre qui lui avait été faite, avant d'inclure l'objet dans un catalogue éloquemment intitulé Faux pharaons à l'occasion d'une exposition qu'il avait consacrée à ce thème à Munich en 1983. Cette même année 1983, un antiquaire zurichois avait reproposé l'œuvre au musée des Arts et Traditions de Genève qui avait, lui aussi, décliné l'offre qui lui était faite. Enfin, dix ans plus tard, la même statue avait été refusée par le comité des experts de la foire des antiquaires de Bâle ${ }^{3}$ lors de laquelle voulait la proposer la galerie Vollmoeller. ${ }^{4}$ C'est donc peu dire que la statue était déjà fortement suspecte lors de sa mise en vente aux enchères. Pourtant, le catalogue de vente rédigé par l'expert Chakib Slitine ne rapportait aucune des péripéties précédentes, alors même que certaines réserves et mises en garde figurent parfois dans ces ouvrages, pas plus qu'il ne donnait d'indication sur l'historique de la statue dont il n'était pas précisé où, quand et par qui elle aurait été découverte. Le catalogue précisait toutefois que la statue portait des hiéroglyphes maladroits qui avaient été poncés de façon rudimentaire et dont la trace apparaissait encore. L'effacement des hiéroglyphes constituait, aux yeux de Dietrich Wildung, un indice supplémentaire de la supercherie. En effet, la connaissance des hiéroglyphes étant longtemps restée imparfaite, les faussaires ont souvent produit des textes incohérents, parfois avec des hiéroglyphes maladroitement tracés. Devant la preuve qui aurait ainsi pu être apportée de la supercherie, l'un des propriétaires aurait pris l'initiative de faire disparaître les hiéroglyphes compromettants, afin de tromper plus facilement un éventuel acquéreur.

C'est sur la base de ces éléments et de la conviction qui en découlait que Dietrich Wildung avait vainement alerté, avant la vente, 
le commissaire-priseur, ainsi que le président de Drouot, en vue de faire retirer l'objet litigieux de la vacation.

L'expert de la vente, Chakib Slitine, s'en tenait à sa propre conviction selon laquelle la statue était authentique et ne voyait pas l'utilité de faire mention de l'avis de Dietrich Wildung dans le catalogue, la personnalité en question fût-elle aussi reconnue que l'éminent spécialiste allemand. Selon l'expert, les inscriptions poncées avaient dû être ajoutées, sans doute au XIX ${ }^{\text {ème }}$ siècle, dans l'intention naïve d'embellir la statue et leur effacement ultérieur n'aurait eu pour conséquence que de rendre à l'objet son état original. Par ailleurs, l'expert - dont la Justice devait ultérieurement souligner le sérieux ayant fait analyser le granit dans lequel est taillé la statue, il ressortait que celui-ci présentait bien toutes les caractéristiques du granit de la région d'Assouan.

Le 17 août 1999, l'avocat des époux Pinault obtenait du Tribunal de Paris en référé, soit dans le cadre d'une procédure d'urgence, la nomination à titre d'experts judiciaires de deux conservateurs du Louvre, Elisabeth Delange, spécialiste de cette période du Moyen Empire au musée du Louvre et conservateur en chef du département des antiquités égyptiennes, et Christiane Desroches-Noblecourt, en retraite après avoir dirigé le même département des antiquités égyptiennes du Louvre de 1973 à 1981. Les deux conservatrices, qui avaient auparavant été contactées par les époux Pinault pour savoir si elles accepteraient d'être désignées comme experts, se voyaient confier la mission de : "dire si la statue de Sésostris III a bien été exécutée sous la XIIème dynastie, à savoir 1878-1843 avant JésusChrist, sinon, dire à quelle date elle a pu être exécutée". Elles devaient, par ailleurs, fournir au tribunal tous les éléments techniques et devaient se faire assister de tout spécialiste, et notamment consulter leurs homologues américains, ainsi que le conservateur allemand par lequel le scandale était arrivé.

La position d'expert judiciaire des deux conservateurs ne pouvait pas manquer d'entraîner des conflits d'intérêts, puisque le Louvre pouvait parfaitement espérer devenir un jour destinataire de 
la statue litigieuse. De plus, circulait déjà le bruit qu'Elisabeth Delange avait été consultée avant la vente et avait émis un avis oral favorable à l'authenticité de la statue (ce fait a d'ailleurs, depuis, été confirmé par Christiane Desroches-Noblecourt (2003), puisqu'elle y écrit: «Madame Pinault, avant la date de la vente, s'était déjà adressée à un conservateur du Louvre, Elisabeth Delange, qui avait émis un avis de première vue favorable»; de même, dans le quotidien Le Monde du 18 août 2003, l'égyptologue Jean Yoyotte rapportait avoir découvert et pu voir la sculpture controversée de Sésostris III avant la vente, en septembre 1998, au cabinet de l'expert Chakib Slitine et que celuici lui avait fait savoir qu'Elisabeth Delange l' avait authentifiée ${ }^{5}$ ). En principe, un expert peut être récusé "s'il a précédemment conseillé l'une des parties". Et, si tel est le cas, il doit le déclarer au juge, comme le stipule l'article 234 du nouveau code de procédure civile. Toutefois, les époux Pinault ne s'opposèrent pas au choix des deux experts et le magistrat ne retint aucune incompatibilité entre les deux fonctions successives d'Elisabeth Delange. Les époux Pinault espéraient-ils alors, en pensant que serait produite une expertise suffisamment convaincante, redonner une virginité à l'œuvre contestée et pouvoir ensuite en faire don au musée du Louvre?

Au terme de six mois d'études, les deux experts ont remis, le 11 avril 2000, un épais rapport de quarante-six pages (hors annexes) dans lequel elles ont récusé chacun des arguments avancés par leur collègue Dietrich Wildung.

Certes, le rapport n'a pas manqué de souligner certaines étrangetés de l'œuvre, difficilement contestables au demeurant. Les experts mentionnent ainsi une "impression d'étrangeté" liée notamment au fait que le style ne correspond pas à l'époque du pharaon représenté. Le visage est qualifié de "déroutant", puisqu'inédit dans la statuaire représentant Sésostris III. Alors que ce souverain, grand chef de guerre, fait généralement l'objet de représentations dramatiques, il est représenté, de façon surprenante, sous des traits apaisés ; pourtant, le doute sur l'identité du souverain représenté n'est pas permis, puisque sous nom figure dans un cartouche. Cette contradiction pourrait sembler de taille, la description 
réaliste des visages constituant une loi qui était "appliquée sans faille" en Egypte pharaonique. D'autres imperfections ne manquent pas de surprendre, notamment les proportions "légèrement" inexactes des jambes par rapport aux canons alors en vigueur. Par ailleurs, le rapport n'a pas tu les problèmes posés par l'absence de toute provenance de l'oeuvre et l'effacement des hiéroglyphes malhabiles. Toutefois, même dans l'antiquité égyptienne, les hiéroglyphes n'étaient pas toujours parfaitement réalisés.

Pourtant, au terme de toutes les restrictions précédentes dont on pourrait légitimement penser que leur conjonction serait apte à faire naître le doute, les experts formulent une thèse audacieuse : les défauts relevés, loin de discréditer la statue, sont en fait tenus pour garants de son authenticité. En effet, un faussaire n'aurait pas - si l'on en croit du moins les auteurs - pu effectuer des erreurs aussi grossières et nombreuses, et s'en serait tenu à toutes les caractéristiques connues de la statuaire de l'époque qu'il aurait voulu pasticher. ${ }^{6} \mathrm{Ce}$ sont, au final, tous les arguments formulés par Dietrich Wildung qui sont contestés et, pour cela, les experts s'en prennent même à l'usage que fait leur collègue allemand à des "références trop restreintes et trop classiques" afin de contester la statue. Pour plaider en la faveur de l'authenticité, les auteurs mettent en avant la beauté de la sculpture, la finesse du détail et du modelé de la musculature. Seule concession faite à la contestation de Dietrich Wildung, le rapport reconnaît qu': "En aucun cas, cette statue ne remonte au règne de Sésostris III (1872-1854 avant notre ère)". Et les experts se risquent à la dater de la fin du Moyen Empire (vers 1850-1720 avant Jésus-Christ), ce qui expliquerait l'inhabituelle douceur des traits du pharaon. Il s'agirait là du seul cas connu de portrait posthume de pharaon, ce qui ne manquerait pas de renforcer encore le caractère exceptionnel de la pièce, les auteurs du rapport attirant l'attention sur ce témoignage historique de grande valeur.

Le rapport remis par les deux experts aurait pu donner satisfaction aux époux Pinault, puisque l'œuvre qu'ils avaient achetée était désormais déclarée authentique. Toutefois, ils décidèrent ensuite, à première vue de façon assez paradoxale, de demander l'annulation 
de la vente. En effet, certaines sources firent état du fait suivant: ayant pris contact, après le dépôt du rapport, avec les dirigeants du musée du Louvre pour savoir si la statue intéresserait leur institution, ils se seraient vu opposer une réponse négative.

Devant le rapport déposé, l'avocat des époux Pinault a pris le parti de s'en remettre à une nouvelle expertise, non plus produite par des conservateurs de musée sur une base stylistique s'appuyant sur les fondements de l'histoire de l'art, mais effectuée par des scientifiques, entendus ici comme spécialistes des sciences exactes. Il a donc demandé qu'un laboratoire spécialisé franco-allemand soit chargé de procéder à une nouvelle expertise, "scientifique" cette fois. Il souhaitait également que soit versé au débat l'avis d'une marchande suisse qui avait fait partie du comité de sélection de la foire de Bâle qui, en 1993, avait refusé d'admettre la statue suspecte en son sein. Il demandait par ailleurs que soit pris en compte l'avis d'un égyptologue américain qui avait pu étudier la statue à New York, lorsqu'elle y fut proposée à la vente. Pour sa part, Dietrich Wildung, reprenant une technique traditionnelle de l'histoire de l'art, proposait de comparer la statue à une vingtaine de portraits royaux de la XIIème dynastie, réunis lors d'une exposition qui se tenait à Würzbug puis à Berlin, et de tenir à cette occasion un colloque d'experts, colloque dont François Pinault se proposait même de prendre en charge les frais. La proposition de Dietrich Wildung visait donc à rendre la parole aux historiens d'art sur la base de la confrontation de visu, une des méthodes les plus légitimes au sein de cette discipline.

Toutefois, les deux experts n'ont pas souhaité revenir sur leur travail et le tribunal les a suivies.

S'en est suivie, en premier jugement, une controverse sur la date exacte de création de l'œuvre. L'avocat des époux Pinault souhaitait obtenir l'annulation de la vente en invoquant le fait que le rapport d'expertise stipulait que la statue avait été sculptée vers 18501720 avant Jésus-Christ, et non pas entre 1878 et 1843 comme cela figurait au catalogue. Sésostris III étant décédé en 1854 avant JésusChrist, l'expert invoqua que la statue pouvait fort bien avoir été créée 
au cours des quatre années séparant son décès du début de la fin du Moyen Empire vers 1850.

Rejetant les arguments avancés par l'avocat des époux Pinault, le tribunal a déclaré la vente parfaite, le 30 janvier 2001, en invoquant que, même si la statue n'a pas été créée du vivant du pharaon représenté, "les caractéristiques de la statue ne sont pas véritablement éloignées de celles du catalogue de vente", étant posé qu' "il doit être entendu une relative imprécision" dans les datations d'une époque aussi lointaine. Se fondant sur le rapport d'expertise qui, étonnament, ne faisait mention des doutes que du seul Dietrich Wildung, les magistrats tranchaient qu' : "il n'existe pas de véritable controverse artistique lorsque seule une personne émet des doutes sur une œuvre", négligeant par là même le fait qu'en histoire de l'art, il est fréquent qu'un seule personne dans le monde fasse autorité pour un artiste donné ou pour une période particulière (Moulin et Quemin, 1993). Et d'attaquer aussi l'impartialité du conservateur allemand en stipulant que: "son excessif acharnement lui fait perdre toute crédibilité".

Etant déboutés en première instance, les époux Pinault ont décidé d'interjeter appel et de changer de mode d'argumentation en invoquant un doute sérieux quant à l'authenticité de la statue. Pour cela, ils ont déposé un rapport rédigé par un jeune égyptologue qu'ils ont mandaté, Luc Watrin, et qui avait envoyé des photographies ${ }^{7} \mathrm{de}$ la statue à de nombreux égyptologues de renom. La plupart avaient répondu en soulignant qu'ils doutaient de l'authenticité de l'œuvre. Les époux Pinault ont également demandé une nouvelle expertise, scientifique, cette fois, pour déterminer l'éventuelle présence sur la statue de particules de métal provenant d'instruments modernes.

Les magistrats semblent s'être formalisés de l'introduction par les époux Pinault d'une requête en nullité de la vente, alors même que l'expertise judiciaire avait déjà décidé qu'il s'agissait d' une statue "parfaitement authentique" et que celle-ci constituait même un "chefd'œuvre unique". Faisant fi du rapport concurrent rédigé par Luc Watrin, la cour d' appel de Paris a tout simplement confirmé le premier jugement. Dans son arrêt du 25 mars 2002, cette cour a donc 
condamné les époux Pinault à payer les 5,1 millions de francs de la vente, augmentés des intérêts (230.000 francs), à assumer les frais d'expertise et à verser un total de 120.000 francs de dommages et intérêts à l'expert qui avait rédigé le catalogue de vente, au commissaire-priseur et au notaire allemand qui avait mis en vente l'œuvre pour le compte d'un de ses clients, en estimant que les époux Pinault savaient qu'il pouvait exister une incertitude sur l'authenticité de l'oeuvre.

Plusieurs égyptologues réputés, en plus de l'expert académique de référence, Dietrich Wildung, tenant pour fausse leur statue de Sésostris III, mais les experts judiciaires la tenant pour authentique, et le tribunal de Paris persistant donc à refuser, sur cette base, l'annulation de la vente, les époux Pinault décidèrent de se pourvoir en cassation, l'affaire devant être plaidée en septembre 2003.

Alors même que l'affaire Sésostris III pouvait sembler close, ${ }^{8}$ François Pinault prit l'initiative de demander au laboratoire FrancineMaurer, spécialisé dans l'étude scientifique et la datation des œuvres d'art, tout particulièrement des objets archéologiques, une analyse de la statue. Sur la base de tests scientifiques, la conclusion qui a été rendue dans le cadre d'un rapport de 20 pages est sans appel: "Cette sculpture est un objet moderne". Le poli de la statue ainsi que la présence de copeaux d'acier fournissent des éléments suffisants pour faire douter la plupart des égyptologues.

Par ailleurs, le 18 août 2003, une figure réputée de l'égyptologie, Jean Yoyotte, professeur honoraire au Collège de France et directeur d'études à l'Ecole Pratique des Hautes Etudes, intervenait, à son tour, dans le débat en accordant une interview (relue et amendée par la personnalité interrogée) au quotidien Le Monde sur l'affaire Sésostris III.

Alors même que, selon la décision judiciaire rendue, la statue controversée devait être tenue pour authentique, Jean Yoyotte n'hésitait pas à remettre en cause cet avis ${ }^{9}$ en soulignant que: "L'autorité des tribunaux français ne saurait en imposer aux membres 
d'une communauté scientifique, par nature transnationale". On voit bien ainsi comment l'égyptologue changeait d'échelle d'appréciation, passant du seul cadre national à l'international, pour mieux remettre en cause le jugement rendu. De même, afin de faire ressortir la différence de compétences entre "experts" en ventes aux enchères et spécialistes en histoire de l'art, il soulignait également: "tout en appréciant l'amitié coopérative des négociants en objets d'art, je sais les limites de la science égyptologique des experts professionnels, fussent-ils agréés près la cour d'appel..." e spécialiste (cf. Trépos, 1996).

Faisant référence au rapport des deux experts désignés par la justice, le professeur honoraire au Collège de France déclarait: "Les arguments avancés dans la discussion proprement typologique m'ont paru souvent fragiles dans l'ensemble, comme dans les détails, et, pour certains, insoutenables". Sans doute afin d'amoindrir l'expertise des deux personnalités consultées, il soulignait que le rapport ne contenait guère que "des données communément reçues qu' on trouve dans tous les manuels". Et de prendre également position contre les manquements à l'éthique de ses deux consoeurs : "La dérive consistant à passer de l'objet de l'expertise à une mise en cause de Wildung est hors de propos et évidemment choquante". En effet, dans son ouvrage Sous le regard des dieux, Christiane Desroches-Noblecourt (2003) affirmait que Dietrich Wildung refusait de reconnaître l'authenticité de la statue de Sésostris III, parce que son maître, Hans Wolfgang Müller, avec lequel ses relations s'étaient déteriorées, la tenait, pour sa part, comme authentique. Par ailleurs, toujours selon Christiane Desroches-Noblecourt, Dietrich Wildung aurait été “vexé parce qu'il ne connaissait pas cette statue quand il a fait sa thèse de doctorat sur les statues du Moyen Empire". Jean Yoyotte poursuivait: "En bonne déontologie, il n'est pas d'usage qu'un chercheur conteste l'honnêteté intellectuelle d'un collègue dont il ne partage pas les conclusions. Les admirables performances de Madame Noblecourt (...) ne l'autorisent pas à transgresser la morale commune". Le professeur honoraire au Collège de France n'hésitait alors pas à retourner le soupçon de concurrence contre l'accusatrice en formulant: "Il faut 
dire au demeurant que Wildung a été un brillant émule et challenger de Christiane Noblecourt".... Jean Yoyotte préférait toutefois évoquer plus longuement les carences du rapport de Christiane DesrochesNoblecourt et Elisabeth Delange en soulignant: "A presque chaque ligne, on trouve des erreurs et des exagérations rhétoriques, des arguments expéditifs et des considérations emphatiques (...). L'exposé des péripéties de l'affaire est aberrant: elle n'aurait commencé qu' "au mois d'août 1999!». Les agissements antérieurs et postérieurs de l'expert sont escamotés (...). Ce texte lâché à la diable souffre de défauts de mémoire et d'information". Par ailleurs, Jean Yoyotte ne manquait pas de faire référence à des éléments scientifiques, mais qu'il replaçait dans un contexte de connaissances archéologiques: 'L'histoire de l'introduction du fer, voire de l'acier en Egypte, fait bon marché des acquis des archéologues en ce domaine".

Jean Yoyotte prenait également ses distances avec le rapport de Luc Watrin: "ses écrits trahissent une problématique vieillotte. Il ignore l'état des questions concernant les 'portraits naturalistes" des rois et use, lui aussi, de la vaine méthode 'anthropométrique'", sans aller toutefois jusqu'à invalider ce travail, puisque: "Son rapport n'en contient pas moins des observations pertinentes. Le 'sondage d'opinion' qu'il a réalisé auprès de savants de différents pays est significatif'.

L'interview de Jean Yoyotte dans Le Monde s'achevait d'ailleurs par la référence faite par l'égyptologue aux analyses scientifiques (entendues ici au sens de sciences dures), puis par un extrait du rapport produit par le laboratoire d'analyses qui avait été sollicité.

Comme le déclarait sans ambage Jean Yoyotte: "Oui (je crois que l'œuvre est moderne). Et je suis loin d'être le seul, comme il ressort de la liste de Watrin, à laquelle on pourrait ajouter quatre antiquaires expérimentés de France et de Suisse et plusieurs égyptologues du Louvre, sans parler des autorités égyptiennes qui ne 
se pressent pas de réclamer la restitution de cette œuvre proclamée de haute importance patrimoniale. Pour moi, la preuve la plus décisive de la falsification est tirée des manœuvres auxquelles s'est livré l'ancien détenteur de la pièce, qui a arasé les inscriptions après que les épigraphistes y ont décelé au premier regard la marque d'un faussaire". S'il achève donc ensuite sa démonstration sur la base d'éléments scientifiques, il ne les tient apparemment pas pour les plus pertinents, "il faut beaucoup de bonne volonté pour accepter l'argument consistant à nier qu'on puisse déceler la différence, pourtant perceptible à l'œil, entre un poli antique et un poli moderne, et à refuser catégoriquement toute crédibilité à l' analyse de laboratoire, quitte à dire plus tard que les sculpteurs égyptiens de l'âge du bronze pourraient bien avoir disposé d'outils en acier!".

L'article du Monde du 18 août 2003 s'achevait sur un paragraphe intitulé "Le verdict des scientifiques", reproduit ci-dessous : "François Pinault a confié, pour analyse, la statue de Sésostris III au laboratoire Francine-Maurer. Voici les résultats de cette analyse : "Le matériau constituant l'objet ne présente pas d'indications d'une altération de longue durée dans un milieu d'enfouissement après la phase de sculpture; l'objet présente des traces d'outils qui correspondent à un façonnage effectué à partir d'outils et d'abrasifs modernes (...); les dépôts présents directement à la surface de la sculpture contiennent des éléments d'origine industrielle. Ces différentes caractéristiques permettent de conclure que cette sculpture est un objet moderne". Par ailleurs, on a découvert des copeaux d'acier inclus dans la statue. Selon Madame Desroches-Noblecourt, rien ne prouve que les Egyptiens ne connaissaient pas les outils en fer sous le Moyen Empire. Le problème est que certains des copeaux sont en acier au chrome, un alliage mis au point au XXème siècle après Jésus-Christ". Pourtant, Christiane Desroches-Noblecourt n'en démord pas. Si l'usage du fer n'était sans doute pas courant pendant le Moyen Empire, nous ne comprendrions, selon elle, toujours pas comment les Egyptiens pouvaient travailler une pierre aussi dure que la diorite et nous ne pouvons pas exclure l'usage d'alliages métalliques... On voit comment la statue, si l'on postule son 
authenticité a priori, serait d'autant plus précieuse qu'elle viendrait nous permettre de renouveler de fond en comble nos connaissances sur une période de l'Egypte antique.

Quelques semaines après Le Monde, Libération publiait à son tour des informations encore plus précises sur les tests scientifiques qui avaient été entrepris, ainsi que sur leurs conclusions. Le laboratoire a effectué un examen de tracéologie, qui consiste à analyser les microparticules adhérant à la pierre, qu'ont pu notamment laisser les outils. Sur une quarantaine de prélèvements sur les différentes parties de la statue, il a trouvé, en plusieurs endroits, des traces d'alliages à base de fer, dont certains, comme le fer-chrome, "n'apparaissent qu'au XX ${ }^{\text {ème }}$ siècle". Les molécules de fer, en outre, ne sont pas corrodées. Le laboratoire pense aussi que les incisions de détails anatomiques ont pu être réalisées à l'aide d'une molette diamantée. Il note la présence de dépôts pigmentaires "blanc jaunâtre" contenant des "éléments d'origine industrielle sans équivoque moderne" ainsi qu'une trace de silice, pouvant provenir "d'un produit de polissage moderne". "Pratiquement toutes les étapes de réalisation de la sculpture montrent l'utilisation d'outils modernes", conclut le rapport, concédant cependant que le martelage aurait pu être exécuté avec des outils en pierre analogues à ceux couramment utilisés au Moyen Empire. Ce qui le conduit à émettre l'hypothèse que le faussaire se serait servi d'un bloc antique".

Un minéralogiste, le professeur Dietrich Klemm, qui a pu étudier la pierre et mener des examens au laboratoire de Pessac, en Gironde, a pu établir que la roche provient d'une carrière de diorite située au Sud d'Assouan, mais que "les traces d'abrasion démontrent sans équivoque l'usage d'un matériel mécanique moderne".

Rebondissement supplémentaire dans l'affaire, Vincent Noce révélait, dans un nouvel article consacré à l'affaire Sésostris III par le quotidien Libération le 10 septembre 2003 que le jeune égyptologue Luc Watrin affirmait même désormais avoir retrouvé le faussaire auteur de l'œuvre contestée dans un atelier du site antique de Memphis et avoir pu filmer ses aveux... 
Pour autant, lors de l'audience de septembre 2003, le parquet a recommandé à la cour de rejeter la demande de révision introduite par les époux Pinault, s'en tenant donc au rapport d'expertise stylistique rédigé par les deux conservatrices du Louvre. D'où l'interrogation qui a pu servir de titre à ce texte: l'art plus fort que la science?

Ce qui pourrait ne constituer qu' un simple fait divers devient le révélateur d'un système tout entier et met à nu l'existence de divers types d'expertise, qui appartiennent à différents registres, mais aussi la possible hiérarchie entre ceux-ci. Interviennent tour à tour en tant qu'experts différents professionnels du monde de l'art, parfois sollicités à des titres divers.

Tel est très nettement le cas d'Elisabeth Delange et de Jean Yoyotte qui, en tant que conservateur de musée pour l'une, professeur d'égyptologie pour l'autre, peuvent formuler des avis experts, mais seulement à titre privé, car ils sont tenus à un devoir de réserve (Moulin et Quemin, 1993). Cependant, après s'en être tenus à un exercice privé de leur expertise, ils basculent dans la sphère publique, la première à la demande des époux Pinault puis de la justice, le second à la probable initiative des époux Pinault. C'est également à la demande de la justice que Christiane Desroches-Noblecourt rompt avec son obligation de réserve et se voit officiellement investie du titre d'expert judiciaire.

Le dimension internationale fait parfaitement ressortir la spécificité du système français d'expertise par les conservateurs de musées et les historiens d'art, puisque leurs homologues étrangers américains ou allemands ne sont pas tenus à une même réserve. C'est d'ailleurs ce qui offre à Dietrich Wildung une grande liberté et lui permet, sur la base de la forte reconnaissance dont il jouit auprès de ses pairs, de se poser en expert.

Un autre type d'expert, Chakib Slitine, est investi de ce titre pour opérer dans le cadre de ventes aux enchères. Le titre d'expert en art n'est, en France, nullement protégé, chacun peut se proclamer expert et apposer une plaque faisant mention de ce titre (Moulin et 
Quemin, 1993). Bien souvent, les experts officiant en ventes aux enchères sont choisis par les commissaires-priseurs - puis par les responsables de sociétés de ventes aux enchères volontaires de biens meubles - pour leur capacité à rassembler les objets qui seront proposés lors de la vacation. Ce n'est donc pas un mystère que la compétence des "experts" en ventes aux enchères est très variable. Le cas rapporté précédemment est toutefois d'autant plus intéressant que Chakib Slitine passe pour sérieux et un chercheur aussi reconnu que Jean Yoyotte ne fait pas mystère que cet expert en ventes aux enchères lui demandait souvent son avis de façon informelle, lorsqu'il avait des doutes sur des objets.

Par ailleurs, un autre type d'experts intervient à travers le bureau d'analyses scientifiques qui n'entre en jeu que tardivement dans l'affaire. On pourrait d'ailleurs fortement s'en étonner si le monde de l'art n'était pas traditionnellement méfiant envers les analyses scientifiques. ${ }^{10}$ Certains expliquent parfois les réticences de la sorte : si l'on systématisait les analyses scientifiques en les appliquant à la plupart des œuvres figurant même dans les plus grands musées, la vague de déclassements qui s'ensuivrait serait probablement de très grande ampleur. Les fondements même de l'histoire de l'art pourraient s'en trouver fortement ébranlés. Ce risque expliquerait pourquoi les conservateurs de musées et historiens d'art seraient, au final, si souvent réticents à faire expertiser les œuvres sur des bases "scientifiques". Ce mouvement irait à l'encontre du souci d'une certaine stabilité qui anime de nombreux conservateurs. ${ }^{11}$

Enfin, un dernier type d'experts intervient finalement à travers les magistrats. Ce sont eux qui, bien que dépourvus de toute compétence spécialisée tant en histoire de l'art ou archéologie qu'en sciences "dures", vont finalement devoir trancher et décider si la statue de Sésostris III est juridiquement authentique, ou s'il ne s'agit que d'un grossier faux moderne.

Par ailleurs, le cas de la statue représentant Sésostris III fait bien ressortir les caractéristiques des deux principaux types d'expertise 
en présence. La première forme, celle que l'on peut qualifier de stylistique ou d'historique, entraîne de forts affrontements entre ses praticiens. Al'inverse, la seconde, celle qui relève des sciences dures, peut produire des jugements tenus pour vrais par l'ensemble de la communauté. ${ }^{12}$

On pourrait penser que le fait de parler d'une seule voix permet alors à ses tenants de faire triompher leurs vues sur celles d'une autre communauté de praticiens, les conservateurs de musées et historiens d'art, aux avis beaucoup plus partagés. Pourtant, il n'en est rien et c'est sur cet apparent paradoxe que nous entendons poursuivre désormais notre réflexion.

\section{Conclusion}

Dans le cadre de ce texte, nous nous sommes arrêté à l'étude d'un seul cas, particulièrement célèbre et emblématique, pour faire ressortir la force des arguments en présence. Le cas de la statue du pharaon Sésostris III est à la fois exemplaire et extrêmement rare. Il faut, en effet, que le justiciable soit aussi fortuné que les époux Pinault pour pouvoir engager les frais liés à un procès en première instance, puis en appel et, enfin, en cassation, mais aussi pour faire diligenter d'autres expertises en plus de celle demandée au tribunal, à la fois auprès d'un jeune archéologue et auprès d'un laboratoire d'analyses scientifiques. C'est précisément parce que les avis experts recueillis sont aussi nombreux que le cas analysé ici fait apparaître à la fois les débats au sein d'une discipline, l'histoire de l'art, mais aussi les arbitrages qui doivent être rendus entre disciplines concurrentes.

Il conviendrait, bien sûr, avant de procéder à toute tentative de généralisation et pour progresser ainsi sur la voie de la théorisation, d'étudier avec autant de minutie d'autres cas qui ont pu défrayer, de façon similaire, la chronique, comme celui du Kouros du musée Getty, mais parfois également judiciaire, comme celui du Jardin à Auvers de Vincent Van Gogh. A chaque fois se rencontrent des mondes sociaux qui, en-dehors de ces cas de controverses, n'entretiennent que peu de contacts et se joue notamment la prééminence d'une 
discipline par rapport à une autre mais aussi, de façon liée, celle d'un monde social par rapport à un autre.

\section{Notes}

1 Sur la rencontre des mondes de l'art et du droit, cf. Heinich (1996), ainsi que Edelman et Heinich (2002).

2 Pour l'anecdote, François Pinault avait demandé que lui soit livrée au plus vite la statue, afin que celle-ci soit présente dans son hôtel particulier du VI ${ }^{\grave{m}}$ e arrondissement de Paris à l'occasion d'une invitation à dîner du président de la République Jacques Chirac, qui avait lieu le soir même de la publication de l'article de Libération...

3 Déjà auparavant, la statue semble avoir beaucoup voyagé. Selon Jean Yoyotte, égyptologue et professeur honoraire au Collège de France, la sculpture aurait été vue durant les années 1970 chez un marchand du Caire, puis chez un marchand de Medinet el-Fayoum. On pourrait supposer que l'œuvre aurait quitté clandestinement l'Egypte et abouti dans un des ports francs de Suisse. Proposée par une galerie zurichoise, elle a été présentée à divers musées en Suisse, en Allemagne et aux Etats-Unis. Tous ont refusé l'offre (Le Monde, lundi 18 août 2003, p. 16).

4 Selon Christian Bessy et Francis Chateauraynaud dans Experts et faussaires (1995, p. 16), "Un faux est réussi s'il parvient à se glisser dans un espace de circulation sans que cela gêne les échanges: il est conforme à une représentation commune et donc à une certaine économie de la perception".

5 Toutefois, étant donné le sentiment d'étrangeté ressenti face à la statue, Jean Yoyotte avait conseillé à l'expert de consulter l'autorité en la matière, Dietrich Wildung. Ceci étant fait, Wildung n'avait pas manqué d'exposer à l'expert de la vente pourquoi il tenait la statue pour fausse, ce pour quoi il avait refusé l'offre d'achat qui lui avait été présentée quelques années plus tôt.

6 'L'objectif d'un faussaire est de répondre à des attentes : son acte suppose qu'une communauté d'acteurs partage un ensemble de préoccupations ou d'objets. Il prend appui sur des représentations communes, sur un espace de calcul partagé" (in Bessy et Chateauraynaud, 1995, p. 227). 
7 Même si cela peut surprendre le néophyte, il est très fréquent de procéder à des expertises sur la base de simples photographies, sans être mis en contact direct avec l'œuvre sur laquelle un jugement sera formulé.

8 Christian Bessy et Francis Chateauraynaud dans Experts et faussaires (1995, p. 201-202), notent ainsi: "une clôture est toujours fragile, puisqu'elle dépend de la stabilité des états des personnes et des choses qui l'ont rendue possible. (...) Le chaînage des épreuves est tellement dense que la controverse, la critique ou le doute peuvent naître à chaque point. C'est pourquoi des objets, jusqu'alors stabilisés, peuvent rebasculer dans l'incertitude".

9 Signalons toutefois que son successeur à la chaire d'égyptologie du Collège de France, Nicolas Grimal, tient, quant à lui, la statue de Sésostris III pour authentique.

10 Dans sa thèse en cours sur les restaurateurs d'œuvres d'art, Léonie Hénault fait état des mêmes réticences d'une autre activité traditionnelle des mondes de l'art face à l'introduction de méthodes scientifiques qui viennent bouleverser son mode d'exercice. Ainsi, le laboratoire du Musée du Louvre n'a été créé qu'en 1931 et a toujours vu ses attributions assez étroitement encadrées.

11 Pierre Bourdieu et Alain Darbel dans L'amour de l'art (1969), soulignaient, au cours des années 1960, que les conservateurs méritaient bien leur nom. Dès lors, il n'y a rien d'étonnant à ce que ces derniers soient peu enclins au changement que signifie le recours à de nouvelles techniques, mais aussi aux nombreuses modifications d'attributions qu'elles sont susceptibles d'entraîner, d'autant plus si ces techniques viennent rivaliser avec leur discipline et remettre en cause le caractère scientifique de celle-ci.

12 Jean-Claude Passeron, dans Le raisonnement sociologique (1991), a parfaitement souligné les différences entre les divers types de sciences, notamment les sciences nomologiques, et ce que cela entraîne au niveau de l'expertise et de l' "administration de la preuve" en histoire de l'art et en sciences exactes. 
Resumo: A arte é mais forte do que a ciência? O confronto entre expertise de estilo e expertise de ciência em uma querela sobre a autenticidade de uma obra de arte: o caso Sésostris III

Uma das dimensões da expertise artística consiste em se pronunciar sobre a autenticidade das obras e, por isso, os experts dispõem de diferentes métodos e recursos. Se, tradicionalmente, o essencial dos argumentos provinha do registro estilístico, da maneira de execução que permitia atribuir esta ou aquela obra a um artista, depois, os novos métodos científicos (testes químicos, de termoluminiscência...), baseados no estudo das propriedades físicas das obras, conheceram um desenvolvimento considerável. Este texto propõe-se a analisar um caso exemplar de controvérsia sobre a autenticidade de uma obra, uma estátua do faraó egípcio Sésostris III, que deu causa a um confronto entre argumentos estilísticos e argumentos científicos (aqui entendidos no sentido de ciências "duras") e estudar como os argumentos opostos foram recebidos por terceiros, como a Justiça, às vezes convocados in fine para solucionar os casos mais delicados.

Palavras-chave: controvérsia arte-ciência-direito, expertise.

\section{Bibliographie}

BECKER, Howard S. Les mondes de l'art. Paris: Flammarion, 1988.

BESSY, Christian; CHATEAURAYNAUD, Francis. Experts et faussaires: pour une sociologie de la perception. Paris: Métailié, 1995.

BOURDIEU, Pierre; DARBEL, Alain. L'amour de l'art: les musées d'art européens et leur public. Paris: Minuit, 1969.

DESROCHES-NOBLECOURT, Christiane. Sous le regard des dieux. Paris: Albin Michel, 2003.

EDELMAN, Bernard; HEINICH, Nathalie. L'art en conflits: l'œuvre de l'esprit entre droit et sociologie. Paris: La Découverte, 2002.

HEINICH, Nathalie. C'est un oiseau! Brancusi vs Etats-Unis, ou quand la loi définit l'art. Droit et société, n. 41, 1996.

HÉNAULT, Léonie. Les restaurateurs d'œuvres d'art. Thèse de doctorat de sociologie en cours, université de Paris-VIII. 
NOCE, Vincent. Descente aux enchères: les coulisses du marché de l'art. Paris: Jean-Claude Lattès, 2002.

MOULIN, Raymonde; QUEMIN, Alain. La certification de la valeur de l'art: experts et expertises. Annales ESC, p. 1421-1445, décembre 1993.

PASSERON, Jean-Claude. Le raisonnement sociologique. Paris: Nathan, 1991.

TRÉPOS, Jean-Yves. La sociologie de l'expertise. Paris: PUF, 1996. (Que sais-je?). 